\title{
The Validity of Salivary Gland Scintigraphy in Sjögren's Syndrome Diagnosis: Comparison of Visual and Excretion Fraction Analysis
}

\author{
María García-González ( $\nabla$ margagon23@hotmail.com ) \\ Hospital Universitario de Canarias https://orcid.org/0000-0001-7600-5308 \\ María Jesús González Soto \\ Hospital Universitario de Canarias \\ María Ángeles Gómez Rodríguez-Bethencourt \\ Hospital Universitario de Canarias \\ Iván Ferraz-Amaro \\ Hospital Universitario de Canarias
}

Research article

Keywords: salivary gland scintigraphy, Sjögren's syndrome, diagnostic value

Posted Date: April 24th, 2020

DOI: https://doi.org/10.21203/rs.3.rs-24082/v1

License: () (i) This work is licensed under a Creative Commons Attribution 4.0 International License. Read Full License

Version of Record: A version of this preprint was published at Clinical Rheumatology on October 19th, 2020. See the published version at https://doi.org/10.1007/s10067-020-05462-0. 


\section{Abstract}

Introduction. The diagnostic value of salivary gland scintigraphy (SGS) in Sjögren's syndrome (SS) is not completely known. Whether qualitative or quantitative methods of SGS interpretation are the most appropriate remains a matter of debate. We sought to determine whether the diagnostic discrimination of quantitative excretion fraction is higher compared to SGS qualitative visual analysis in a cohort of subjects with suspected SS.

Materials and methods. Cross-sectional study that encompassed 204 subjects who underwent SGS for potential SS diagnosis. Based on clinical judgement, three groups were established: SS, non-SS autoimmune diseases (AID non-SS) and neither SS nor other AID (non-AID). In addition, American-European Consensus Group -AECG- and American College of Rheumatology-ACR- criteria were applied. Qualitative diagnosis through visual analysis -normal vs. abnormal and Schall's classification grade- and semiquantitative and quantitative excretion fraction (EF\%) scores were established following SGS assessment. The diagnostic discrimination of the different SGS scores for the various SS diagnostic modalities (clinical judgement and AECG and ACR criteria) was compared through their areas under the curve (AUC).

Results. Most SGS parameters were significantly associated with SS-related clinical and laboratory features. Schall's grade $\geq$ III was significantly more frequent in SS than in non-SS patients. In general, EF\%-derived parameters did not show significant differences between groups. AUC of Schall's classification reached statistical significance in its diagnostic discrimination for SS clinical judgement (AUC 0.704 [95\% Cl 0.597-0.811], $\mathrm{p}=0.000)$ and AECG criteria (AUC 0.764 [95\% Cl $0.641-0.886], p=0.000$ ). Similarly the EF\% submandibular mean (AUC 0.737 [95\% $\mathrm{Cl} 0.546-0.931] \mathrm{p}=0.032$ ) was significantly associated with SS diagnosis through ACR criteria. However, AUC comparisons between qualitative and quantitative methods did not yield significant values.

Conclusion. SS diagnostic discrimination of EF\% is not superior to that obtained by qualitative visual analysis.

\section{Introduction}

Sjögren's syndrome (SS) is a chronic autoimmune inflammatory disorder characterized by exocrine glandular tissue involvement. SS has therefore been considered an autoimmune epithelitis in which the main target is salivary glands (1). Xerostomia is the most common clinical manifestation of salivary gland involvement in SS (2). However, xerostomia is a nonspecific symptom with a prevalence that ranges from 12 to $30 \%$ in the general population (3). Moreover, xerostomia correlates poorly with glandular involvement (4) and, in many cases, it does not stem from parenchymal damage (xerostomia vera) but rather from psychogenic or pharmacological causes (xerostomia spuria) (3). Besides, xerostomia vera is a symptom that typically manifests late during the disease, as it can go unnoticed until a $50 \%$ decrease of saliva secretion volume has occurred (3).

Among the currently accepted techniques to evaluate salivary gland involvement in SS, salivary gland scintigraphy (SGS) addresses it from a functional perspective. It is the only method that facilitates identification of cases with xerostomia vera and that provides information regarding the distribution and severity of the glandular dysfunction in the four major glands all in a single procedure (4). However, SGS qualitative interpretation based on the visual analysis proposed in the AmericanEuropean Consensus Group (AECG) criteria for SS (5) has been criticized (6). Its subjective interpretation and the procedure's lack of standardization make it an observer-dependent technique, with only moderate degrees of concordance in previous reports (7). In an attempt to overcome this limitation, different quantitative indices derived from SGS time-activity curves have been proposed. However, those studies that have analyzed them are very heterogeneous, limiting any generalization of their conclusions (8-16). Although no consensus has been reached regarding which indices would be more appropriate in the SS diagnosis, some authors have suggested that those based on excretion would be the most useful during the early stages of the disease, particularly the percentage stimulated excretion fraction (EF\%) $(6,13)$. 
In the present study, we aimed to determine if the EF\% diagnostic discrimination is higher compared to that of the SGS visual analysis in a cohort of subjects with suspected SS. We additionally sought to establish whether SGS is related to clinical and laboratory features of SS.

\section{Materials And Methods}

\section{Subjects}

In this study, all patients who had been referred to the Rheumatology Division of the Hospital Universitario de Canarias due to suspected SS between 2006 and 2012 and who had undergone SGS were selected. Recruitment was halted in 2012 when the American College of Rheumatology (ACR) published its classification criteria for SS, in order to minimize selection and/or indication biases. These criteria did not take SGS into account, but only salivary gland biopsy when assessing oral involvement (17), a fact that might have influenced rheumatologists. Two hundred and seventy-two patients had undergone an SGS at the Nuclear Medicine Division of the Hospital Universitario de Canarias during that time frame. Fifty-seven cases not referred due to suspected SS, 11 patients who had already been diagnosed with SS, and 9 whose medical data were not available were excluded. Of the 195 patients included, most had been referred to a rheumatologist because of dryness symptoms (28\%), musculoskeletal manifestations (21\%) or abnormal laboratory findings (24\%). Other less frequent causes were skin manifestations (7\%), salivary glandular alterations (3\%), ocular symptoms (3\%), and other features that from the primary care physician's point of view were suspicious of connective tissue diseases (6\%). After SGS was performed, patients were evaluated under standard clinical practice conditions to determine if the SS diagnosis was applicable. Patients were therefore categorized as SS or non-SS. According to the rheumatologist's clinical judgment, 81 patients were diagnosed with SS (52 primary forms, 23 secondary forms and 6 probable forms). The diagnosis among the non-SS subjects were as follows: other autoimmune diseases (28 undifferentiated forms (18), 18 defined forms, and 12 organ-specific forms), 28 non-autoimmune diseases, and 17 cases with non-specific manifestations. In the remaining 11 subjects diagnostic data were not available.

\section{Data acquisition}

Information was collected by reviewing patient records, using the SGS date as the time reference: i) demographic and medical data, including conditions listed as exclusion criteria in the AECG (5) and/or the ACR (17) SS classification criteria, sicca symptoms and salivary gland swelling and their evolution time, dry-mouth and/or salivary dysfunction-related diseases and drugs $(19,20)$, and clinical diagnosis; ii) laboratory data: ANA (HEp-2) and its specificities (ENA) (Western-blot), double-stranded anti-DNA antibodies (Crithidia Iucilliae and ELISA), rheumatoid factor, gammaglobulinemia and immunoglobulins, erythrocyte sedimentation rate, complement levels, hepatitis B, C and HIV serology; iii) Schirmer I test, in vivo ocular stains and lip salivary gland biopsy (obtained by a 4-mm punch incision (21) and graded according to the Chisholm and Mason scoring system (22)); iv) SS classification criteria fulfillment: AECG (5), ACR (17), and an AECG criteria variant, which requires the same score to be classified but does not allow use of SGS component. This was consistent with the methods similarly applied by other authors $(11,12)$. Medical data on ocular evaluation were accessible in 132 cases. Lip salivary gland biopsy was performed in 71 patients. The median time between these two tests and the SGS was 1 (interquartile range [IQR] -2-9) and 2 (IQR - 1-5) months, respectively. Of the 137 patients with available data on EF\%, AECG criteria was fulfilled in 81 subjects (38 primary SS, 8 secondary SS and 35 non-SS). Non-SGS AECG criteria variant was fulfilled in 74 subjects and the ACR criteria in 32 (15 primary SS, 17 non-SS). Insufficient data prevented classification in the remaining cases.

\section{SGS assessment}

SGS was performed with the patient in the supine position and Water's projection after intravenous injection of $370 \mathrm{MBq}$ $(10 \mathrm{mCi})$ of ${ }^{99 \mathrm{~m}} \mathrm{Tc}$. A single-headed gamma camera (Philips-Adac Vertex Plus Epic) was used with parallel front and rear collimators. Images were collected onto a $64 \times 64$ pixels matrix at 1 second/frame during the first minute and 2 seconds/frame during the remaining 29. In the 15th minute salivary excretion was stimulated by administering $10 \mathrm{ml}$ of 
lemon juice. Regions of interest were manually drawn on summed dynamic images: one for each parotid and submandibular gland and one on the cranial vault as the background reference. Once the background activity was subtracted, time-activity curves were generated, from which EF\% was obtained. EF\% refers to the saliva percentage contained in the salivary glands that is excreted into the oral cavity in response to the stimulus (100x[1-(minimum poststimulus net counts/maximum pre-stimulus net counts)]) (23). Qualitative diagnosis, normal vs. abnormal and Schall's classification grade, was assessed through visual analysis. Schall's classification, as previously described, consists of: grade I (normal uptake, concentration and excretion of the tracer), grade II (mild to moderate delayed and/or reduced uptake and/or concentration of the tracer), grade III (markedly delayed and reduced concentration and excretion of the tracer) and grade IV (complete absence of an active concentration) $(4,24)$. In addition, EF\% values for each gland were used to calculate parotid, submandibular and overall glands means and the semi-quantitative index score proposed by Shizukuishi (14) and other authors (25). No patients had undergone radiotherapy and/or chemotherapy prior to the SGS assessment. Data on 4 submandibular glands (3 right-sided and 1 left-sided) were not obtainable due to sialectomy, and in one patient data on both parotids were missing. Schall's classification grade was determined in 147 cases. EF\% values were available in the Nuclear Medicine reports of 137 of the 195 cases included in the study. Those were the cases analyzed for statistical purposes.

\section{Statistical analysis}

Demographic and clinical characteristics of patients with SS and non-SS subjects were described as mean \pm standard deviation or percentages for categorical variables. For non-normally distributed continuous variables, data were expressed as a median and IQR. Univariate differences between patients and controls were assessed through T Student, U MannWhitney, Chi squared or Fisher Exact tests according to normal distributions or number of subjects. Associations between different SGS parameters and SS-related features were analyzed through logistic and linear univariable regressions. SGS diagnostic discrimination with respect to SS diagnosis was analyzed by the area under the curve (AUC). Differences between AUCs were calculated using the DeLong method (26). Cut-off points for EF\% were selected by calculating the Youden index. Diagnostic efficiency through sensitivity (S), specificity (E) and positive likelihood ratio (LHR+) were determined for each SGS parameter. All the analyses used a 5\% two-sided significance level and were performed using SPSS software, version 21 (IBM, Chicago, IL, USA) and STATA software, version 15/SE (Stata Corp., College Station, TX, USA). A $p$ value $<0.05$ was considered statistically significant.

\section{Results}

\section{Cohort characteristics}

Table 1 shows the differences between the SS and non-SS patients analyzed in our study. In summary, SS and non-SS patients were comparable in terms of sex, age, and current smoking but most sicca syndrome-related data were more frequently observed in SS patients. In this sense, although dry-mouth and dry-eye symptoms were not more prevalent in SS patients, major salivary gland swelling $(63 \% v s .20 \%, p=0.009)$, a positive lip salivary gland biopsy $(57 \% v s 13 \%, p=0.001)$, and a positive ocular test $(83 \%$ vs. $55 \%, p=0.004)$ were more common in patients with SS. Only $15 \%$ of the patients with xerostomia had been referred more than one year since onset, with no evident differences between SS and non-SS patients $(10 \%$ vs. $18 \%, \mathrm{p}=0.27)$ (data not shown).

Regarding xerostomia-related comorbidities, no differences were found in thyroid gland disease, other organ-specific autoimmune diseases, and other dry-mouth related diseases or dry-mouth related drugs. However, patients with SS experienced more non-SS systemic autoimmune diseases $(28 \%$ vs. $11 \%, p=0.001)$. Lastly, as might have been expected, patients with SS more frequently exhibited laboratory abnormalities such as the presence of rheumatoid factor, hypergammaglobulinemia, ANA positivity, and the presence of both anti-SSA/Ro and anti-SSB/La antibodies.

\section{Salivary gland scintigraphy results}


The differences in SGS results between SS and non-SS patients are shown in Table 2. In this analysis, non-SS patients were divided in non-SS autoimmune diseases (AID non-SS) and neither SS nor other AID (non-AID) subjects. Accordingly, comparisons were made between SS and AID non-SS, and between SS and non-AID groups. Interestingly, in all groups, both the submandibular and parotid glands were dysfunctional in most patients. Dysfunction of just one glandular type, submandibular or parotid, was more frequent in the non-AID than in the AID non-SS group $(45 \% v s .24 \%, p=0.20)$ and in the non-AID than in the SS group ( $45 \%$ vs. $26 \%, p=0.15)$, although statistical significance was not reached (data not shown).

Regarding qualitative visual analysis, SGS was more frequently abnormal in SS patients compared to the other groups. In all groups, uptake dysfunction was more common than excretion dysfunction. Specifically, all patients with SS showed uptake dysfunction. Furthermore, severe forms (Schall's grade $\geq$ III) were more common in SS patients and were significantly more frequent than in the non-SS AID $(61 \%$ vs. $21 \%, \mathrm{p}=0.000)$ and in the non-AID $(61 \% v s .17 \%, \mathrm{p}=0.000)$ groups.

Concerning EF\%-derived parameters, although SS patients presented, in general, lower EF\% values, differences were only significant in the comparison of SS and non-AID groups for the right submandibular gland (27 [IQR 11-41] vs. 43 [IQR 26-

$48] \%, p=0.014$ ) and for the EF\% submandibular mean (30 [IQR 15-44] vs. 40 [IQR 26-52] \%, $p=0.030$ ). No differences were found in the remaining comparisons between groups in other EF\% gland values. Regarding semi-quantitative index scores, patients with SS disclosed a higher value (4 [IQR 2-6] vs. 3 [IQR 1-5] points, $\mathrm{p}=0.026)$ in the submandibular score. Although a trend was found, no difference was statistically significant in the overall glands score comparison between patients with SS and non-AID subjects (6 [IQR 2-9] vs. 4 [IQR 1-8] points, p = 0.13). .

\section{Association between SGS parameters and Sjögren's syndrome-related features}

Remarkably, the presence of dry-mouth and dry-eye symptoms and positive ocular tests were not associated with any of the SGS analysis modalities. Lip salivary gland biopsy disclosed a trend in its relation with EF\% semi-quantitative index scores although, in this case, statistical significance was not reached. Submandibular EF\%-derived parameters were associated with ANA $\geq 1 / 320$ (beta coef. 1.12 [95\% Cl 0.29-1.92] points, $p=0.008$ ), anti-SSA/Ro antibodies (beta coef. 0.83 [95\%Cl $0.02-1.64$ ] points, $p=0.046$ ), rheumatoid factor (beta coef. 1.14 [95\% $\mathrm{Cl} 0.35-1.94]$ points, $p=0.005$ ), hypergammaglobulinemia (beta coef. 0.98 [95\% $\mathrm{Cl} 0.19-1.77$ ] points, $p=0.016$ ), and major salivary gland swelling (beta coef. 1.38 [95\% Cl 0.13-2.63] points, $p=0.031$ ). Similarly, the overall glands semi-quantitative index score was associated with major salivary gland swelling. Among variables derived from visual analysis, Schall's grade $\geq$ III reproduced the same associations as the submandibular EF\%-derived parameters, except for major salivary gland swelling (Table 3).

\section{Salivary gland scintigraphy data in discriminating patients with Sjögren's syndrome}

Table 4 shows the receiver operating curve (ROC) analyses of different SGS modalities according to various SS diagnostic criteria. In this sense, the AUCs for SGS visual analysis, both for presence of abnormalities and Schall's classification, reached statistical significance in their discrimination for the SS clinical judgement (Schall's classification AUC $0.704[95 \% \mathrm{Cl}$ 0.597-0.811], $p=0.000$ ) and AECG criteria (Schall's classification AUC 0.764 [95\% Cl 0.641-0.886], $p=0.000$ ). However, no statistical significance was found for the non-SGS AECG criteria variant, nor for the ACR criteria when this visual analysis was considered. AUCs for the AECG criteria were significant for all the SGS analysis modalities. However, these associations were lost when the analysis was performed with the non-SGS AECG criteria variant. Interestingly, for the ACR criteria, which do not encompass an SGS-related component in their construction, AUCs were significant in the analysis for submandibular semi-quantitative index score (AUC 0.723 [95\% $\mathrm{Cl} 0.524-0.922], p=0.045$ ) and the EF\% submandibular mean (AUC 0.737 [95\% Cl 0.546-0.931], $\mathrm{p}=0.032$ ) (Table 4). When comparisons were made between AUCs in the AECG criteria diagnostic modality, taking Schall's classification as the reference category, no statistical differences were found between qualitative and quantitative SGS methods. 
Cut-off points were obtained from the ROC curves of the AECG criteria diagnostic modality. In this sense, submandibular EF\% mean had a cutoff of $<38 \%$ (S 73\%, E 59\%, LHR +1.78) and submandibular semi-quantitative index score a cutoff of $\geq$ 4 units (S 67\%, E 66\%, LHR + 1.97). Schall's grade $\geq I I I$ had an S, E and LHR + of, respectively, 68\%, 84\%, and 4.25 .

\section{Discussion}

During the last decade, the use of SGS in SS diagnosis has continuously declined (2). At the same time, there has been an explosion in other techniques such as ultrasound (27), and several authors have proposed its use to replace SGS $(28,29)$. However, unlike ultrasound, which evaluates glandular involvement from a morphological perspective, SGS is a functional technique. This implies differential capabilities in the diagnosis of SS such as identification of xerostomia vera cases and detection of glandular dysfunction forms due to neurotransmission blockade, and not only because of glandular damage (6, 10). In addition, SGS is able to detect dysfunction from the moment that $25 \%$ of the parenchyma becomes involved, when xerostomia may have not been noticed (4). However, SGS visual interpretation has been criticized for its subjectivity (6), and the use of quantitative indices has not been sufficiently supported by the studies carried out to date (8-16). According to our results, SGS is a test boasting a high relation to laboratory and pathophysiological features of SS, which supports its validity as a diagnostic tool in this disease. However, its interpretation using a quantitative, versus a qualitative, method did not yield significant differences in terms of diagnostic discrimination.

In our study, in line with previous reports, SS patients exhibited predominantly submandibular involvement. Moreover, considerable overlapping in EF\% values between groups was noted (8-10, 15, 30-32). In addition, EF\% results were not different between groups except for those relating to the right submandibular values. This is in accordance with a report by Nishiyama et al. (11), who found no differences in parotid EF\% between SS and controls, and with the studies by Infante et al. (30) and Kim et al. (33), in which asymmetrical involvement with right-sided predominance was also found. We agree with the fact that, as described by these authors, random probability might explain this uneven involvement. However, we would additionally suggest that anatomical asymmetry concerning irrigation, drainage and autonomic innervation could play a role in the right-sided predominance found in our own study.

Although other reports have found differences in EF\% between SS and controls $(9,12,14)$, it should be noted that those reports frequently used as comparators only healthy subjects or subjects affected by non-autoimmune sicca syndrome, and did not include patients affected by other AIDs. Moreover, these reports excluded subjects who met the exclusion criteria of the AECG classification criteria for SS, a fact that implies the exclusion of conditions that frequently mimic SS in its clinical presentation. We additionally believe that selection of severe cases may explain the differences observed between SS and non-SS patients in other studies. For example, in a recent retrospective study by Kaldeway et al. (31), differences were found in EF\% values for the four glands between SS and non-SS patients. This study only included subjects who had undergone all the necessary tests to be classified according to AECG criteria. Moreover, while the mean EF\% values in our work were similar to those observed by Aksoy et al. (10), those of the study by Kaldeway et al. were considerably lower (31). We believe that our results may be more accurate given that our approach was to include a real cohort of subjects clinically suspected to have SS, and not just healthy subjects or non-autoimmune sicca syndrome patients.

In our study we found an association between submandibular EF\%-derived parameters, and a Schall's grade $\geq$ III, with serological markers of B-lymphocyte activity. This observation is consistent with current knowledge about the pathophysiology of glandular damage in SS, which would involve exposure to certain autoantigens in the epithelium and chronic activation of B-lymphocytes $(1,34)$. Our results agree with a previous study involving a large retrospective cohort of primary SS that found an association of Schall's grade IV with elevated ANA titers, rheumatoid factor, anti-SSA/Ro antibodies, and complement consumption (35). In contrast to two previous reports, we found association of EF\% with antiSSA/Ro antibodies and immunoglobulins $(12,31)$. Other studies have focused on the association between SGS and salivary gland biopsy. In this sense, results have been contradictory. For example, while in two reports a correlation was found between the Chisholm and Mason score and EF\% (10) and between EF\% values and focus score (9), in another study this 
association was not evident (31), as occurred in our sample. Nevertheless, due to the fact that our study linked serological typical findings of the disease to all of the modalities pertaining to SGS analysis, we believe our results support its use as a diagnostic test that correlates with the pathophysiology of the disease.

Our results of AUC, S and E for submandibular EF\% parameters are higher than those found by Infante et al. (30), although the cut-off points established in both cases are similar. Moreover, our findings are in agreement with those reported by Kim et al. (33), who established much higher cut-off points. The characteristics of the subjects included in each study and the prevalence of SS in each sample, as well as the use of static -instead of dynamic- SGS images in the latter study, could explain this variability. In a study by Vinagre et al., a Schall's grade $\geq$ III yielded an S and E of 75\% and 78\%, respectively, compared to the $68 \%$ and $84 \%$ figures obtained with our sample (36). In the study by Infante et al., although S reached $89 \%$ and E $100 \%$, discrimination was calculated between SS patients and healthy subjects, and not between SS patients and other forms of sicca syndrome and/or autoimmune diseases (30). In any case, and in line with what has been identified by Kim et al (33), the best performances of the EF\%-derived parameters in our sample were only moderate and their efficiencies, once the cut-off points were established, were not superior to that of a Schall's grade $\geq$ III. Therefore, our data debilitate the application of EF\% for diagnosing SS patients in a real-world clinical practice setting.

The main limitation of our study is its retrospective design. However, we believe that the selection of incident cases and the fact that neither sialography nor sialometry are performed for SS evaluations in our center has minimized selection biases. Some of the missing data in the Schall's classification parameter in our study may not have been random, but rather reflect the difficulty of choosing one or another Schall's grade by the nuclear physician in doubtful cases. Moreover, although EF\% values were not available for rheumatologists at the time of diagnosis, SGS visual analysis findings were. This fact may have overestimated our diagnostic performance results for the visual analysis modalities of SGS in the "clinical judgement" and "AECG criteria" diagnostic modalities. However, the impact of partial verification bias and circular reasoning, a common limitation in previous studies of diagnostic tests in SS (37), has been minimized in our study, since other diagnostic modalities that do not include SGS in their construction were also used. For instance, as previously mentioned, we constructed and used as a diagnosis modality AECG criteria without the SGS component and the ACR criteria.

\section{Conclusions}

In our study, Schall's grade and submandibular EF\%-derived parameters were associated with B-lymphocyte activity markers in patients with suspected SS. This reinforces the concept that SGS assessment may be related to the pathophysiology and prognosis of SS. However, EF\%-derived parameters, probably due to their high dispersion and the difficulty of establishing an appropriate cut-off point, are not superior to that of SGS visual analysis when diagnosing SS. Today, the diagnosis of SS continues to be a challenge for the clinician. More studies are needed to further elucidate the role of SGS in patients with SS.

\section{Abbreviations}

ACR: American College of Rheumatology

AECG: American-European Consensus Group

AID: autoimmune diseases

ANA: antinuclear antibodies

AUC: area under the curve

Cl: confidence interval

coef: coefficient 
E: specificity

$E F \%$. percentage stimulated excretion fraction

IQR: interquartile range

LHR+: positive likelihood ratio

OR: odds ratio

ROC: receiver operating curve

S: sensitivity

SS: Sjögren's syndrome

SGS: salivary gland scintigraphy

\section{Declarations}

\section{Acknowledgments}

We thank the Sociedad Española de Reumatología for its assistance in the English-language review of this manuscript.

\section{Authors' contributions}

IFA and MGG designed and performed the research, analyzed data, and wrote the manuscript. MJGS and MAGRB acquired the SGS images and helped interpreting data, and assisted in preparing the manuscript. All authors read and approved the final manuscript.

\section{Funding}

Not applicable.

\section{Availability of data and materials}

Data are available from the corresponding author on a reasonable request.

\section{Ethics approval and consent to participate}

This study was approved by the institutional review board of the Hospital Universitario de Canarias (IRB approval number 2014_18) to be in accordance with the ethical standards and with the Helsinki Declaration of 1975, as revised in 2013.

\section{Consent for publication}

Not applicable.

\section{Competing Interests}

The Authors declare that there is no conflict of interest.

\section{References}

1. Brito-Zerón P. Baldini C, Bootsma H. Bowman SJ, Jonsson R. Mariette X, et al. Sjögren syndrome. Nat Rev Dis Primer [Internet]. 2016 [citado 21 de octubre de 2019];2(1). Disponible en: http://www.nature.com/articles/nrdp201647. 
2. Rischmueller M. Tieu J, Lester S. Primary Sjögren’s syndrome. Best Pract Res Clin Rheumatol. 2016;30(1):189-220.

3. Tanasiewicz M. Hildebrandt T, Obersztyn I. Xerostomia of Various Etiologies: A review of the literature. Adv Clin Exp Med. 2016;25(1):199-206.

4. Vinagre F. Santos MJ, Prata A. da Silva JC, Santos Al. Assessment of salivary gland function in Sjögren's syndrome: the role of salivary gland scintigraphy. Autoimmun Rev. 2009;8(8):672-6.

5. Vitali C. Bombardieri S, Jonsson R. Moutsopoulos HM, Alexander EL. Carsons SE, et al. Classification criteria for Sjögren's syndrome: a revised version of the European criteria proposed by the American-European Consensus Group. Ann Rheum Dis. 2002;61(6):554-8.

6. Vivino FB. Hermann GA. Role of nuclear scintigraphy in the characterization and management of the salivary component of Sjögren's syndrome. Rheum Dis Clin N Am. 2008;34(4):973-86.

7. Cortés-Blanco A. Martínez-Lázaro R. [Reproducibility of the qualitative interpretation of dynamic salivary radionuclide scans with excretory stimulation]. Acta Otorrinolaringol Esp. 2000;51(2):143-8.

8. Dugonjić S. Stefanović D, Ethurović B. Spasić-Jokić V, Ajdinović B. Evaluation of diagnostic parameters from parotid and submandibular dynamic salivary glands scintigraphy and unstimulated sialometry in Sjögren's syndrome. Hell $\mathrm{J}$ Nucl Med. 2014;17(2):116-22.

9. Zou Q. Jiao J, Zou M-H. Xu J-H, Pan Y-F. Chen J-N, et al. Semi-quantitative evaluation of salivary gland function in Sjögren's syndrome using salivary gland scintigraphy. Clin Rheumatol. 2012;31(12):1699-705.

10. Aksoy T. Kiratli PO, Erbas B. Correlations between histopathologic and scintigraphic parameters of salivary glands in patients with Sjögren's syndrome. Clin Rheumatol. 2012;31(9):1365-70.

11. Nishiyama S. Miyawaki S, Yoshinaga Y. A study to standardize quantitative evaluation of parotid gland scintigraphy in patients with Sjögren's syndrome. J Rheumatol. 2006;33(12):2470-4.

12. Henriksen AM. Nossent HC. Quantitative salivary gland scintigraphy can distinguish patients with primary Sjøgren's syndrome during the evaluation of sicca symptoms. Clin Rheumatol. 2007;26(11):1837-41.

13. Booker J. Howarth D, Taylor L. Voutnis D, Sutherland D. Appropriate utilization of semi-quantitative analysis in salivary scintigraphy. Nucl Med Commun. 2004;25(12):1203-10.

14. Shizukuishi K. Nagaoka S, Kinno Y. Saito M, Takahashi N. Kawamoto M, et al. Scoring analysis of salivary gland scintigraphy in patients with Sjögren's syndrome. Ann Nucl Med. 2003;17(8):627-31.

15. Adams BK. Al Attia HM, Parkar S. Salivary gland scintigraphy in Sjögren's syndrome: are quantitative indices the answer? Nucl Med Commun. 2003;24(9):1011-6.

16. Aung W. Murata Y, Ishida R. Takahashi Y, Okada N. Shibuya H. Study of quantitative oral radioactivity in salivary gland scintigraphy and determination of the clinical stage of Sjögren's syndrome. J Nucl Med Off Publ Soc Nucl Med. 2001;42(1):38-43.

17. Shiboski SC. Shiboski CH, Criswell LA. Baer AN, Challacombe S. Lanfranchi H, et al. American College of Rheumatology classification criteria for Sjögren's syndrome: a data-driven, expert consensus approach in the Sjögren's International Collaborative Clinical Alliance Cohort. Arthritis Care Res. 2012;64(4):475-87.

18. García-González M. Rodríguez-Lozano B, Bustabad S. Ferraz-Amaro I. Undifferentiated connective tissue disease: predictors of evolution into definite disease. Clin Exp Rheumatol. 2017;35(5):739-45.

19. Saleh J. Figueiredo MAZ, Cherubini K. Salum FG. Salivary hypofunction: An update on aetiology, diagnosis and therapeutics. Arch Oral Biol. 2015;60(2):242-55.

20. Han P. Suarez-Durall P, Mulligan R. Dry mouth: a critical topic for older adult patients. J Prosthodont Res. 2015;59(1):619.

21. Guevara-Gutiérrez E. Tlacuilo-Parra A, Minjares-Padilla LM. Minor salivary gland punch biopsy for evaluation of Sjögren's syndrome. J Clin Rheumatol. 2001;7(6):401-2.

22. Chisholm DM. Mason DK. Labial salivary gland biopsy in Sjögren's disease. J Clin Pathol. 1968;21(5):656-60. 
23. Demangeat R. Didon-Poncelet A, Cherfan J. Demangeat J-L. Stimulated salivary pertechnetate clearance revisited: correlation with dynamic scintigraphic indices in sicca syndrome. Clin Nucl Med. 2000;25(11):888-94.

24. Schall GL. Anderson LG, Wolf RO. Herdt JR, Tarpley TM. Cummings NA, et al. Xerostomia in Sjögren's syndrome. Evaluation by sequential salivary scintigraphy. JAMA. 1971;216(13):2109-16.

25. Milic VD. Petrovic RR, Boricic IV. Marinkovic-Eric J, Radunovic GL. Jeremic PD, et al. Diagnostic value of salivary gland ultrasonographic scoring system in primary Sjögren's syndrome: a comparison with scintigraphy and biopsy. J Rheumatol. 2009;36(7):1495-500.

26. DeLong ER. DeLong DM, Clarke-Pearson DL. Comparing the areas under two or more correlated receiver operating characteristic curves: a nonparametric approach. Biometrics. 1988;44(3):837-45.

27. Baldini C. Zabotti A, Filipovic N. Vukicevic A, Luciano N. Ferro F, et al. Imaging in primary Sjögren's syndrome: the «obsolete and the new». Clin Exp Rheumatol. 2018;36 Supp/112(3):215-21.

28. Milic V. Petrovic R, Boricic I. Radunovic G, Marinkovic-Eric J. Jeremic P, et al. Ultrasonography of major salivary glands could be an alternative tool to sialoscintigraphy in the American-European classification criteria for primary Sjogren's syndrome. Rheumatology. 2012;51(6):1081-5.

29. Salaffi F. Carotti M, lagnocco A. Luccioli F, Ramonda R. Sabatini E, et al. Ultrasonography of salivary glands in primary Sjogren's syndrome: a comparison with contrast sialography and scintigraphy. Rheumatology. 2008;47(8):1244-9.

30. Infante JR. García L, Rayo JI. Serrano J, Domínguez ML. Moreno M. Diagnostic contribution of quantitative analysis of salivary scintigraphy in patients with suspected Sjögren's syndrome. Rev Espanola Med Nucl E Imagen Mol. 2016;35(3):145-51.

31. Kaldeway HP. ter Borg E-J, van de Garde EMW. Habraken JBA, van Buul MMC. Validation of quantitative salivary gland scintigraphy in relation to the American-European concensus criteria for Sjögren's syndrome: Nucl Med Commun. 2019;40(4):343-8.

32. Umehara I. Yamada I, Murata Y. Takahashi Y, Okada N. Shibuya H. Quantitative evaluation of salivary gland scintigraphy in Sjörgen's syndrome. J Nucl Med Off Publ Soc Nucl Med. 1999;40(1):64-9.

33. Kim H-A. Yoon S-H, Yoon J-K. Lee SJ, Jo KS. Lee DH, et al. Salivary gland scintigraphy in Sjögren's syndrome: comparison of the diagnostic performance of visual and semiquantitative analysis. Nuklearmedizin. 2014;53(4):13945.

34. Ferro F. Marcucci E, Orlandi M. Baldini C, Bartoloni-Bocci E. One year in review 2017: primary Sjögren's syndrome. Clin Exp Rheumatol. 2017;35(2):179-91.

35. Ramos-Casals M. Brito-ZeróN P, Perez-De-Lis M. Diaz-Lagares C, Bove A. Soto M-J, et al. Clinical and prognostic significance of parotid scintigraphy in 405 patients with primary Sjögren's syndrome. J Rheumatol. 2010;37(3):585-90.

36. Vinagre F. Santos Al, Santos MJ. Prata A, Oliveira A. Canas da Silva JC. [Salivary gland scintigraphy in the evaluation of patients with sicca complaints]. Acta Reumatol Port. 2008;33(4):422.

37. Guellec D. Cornec D, Jousse-Joulin S. Marhadour T, Marcorelles P. Pers J-O, et al. Diagnostic value of labial minor salivary gland biopsy for Sjögren's syndrome: A systematic review. Autoimmun Rev. 2013;12(3):416-20.

\section{Tables}


Demographics

$\begin{array}{cc}\text { Non-SS } & \text { SS } \\ (n=83) & (n=54)\end{array}$

Women, n (\%)

$80(96) \quad 53(98) \quad 1.00$

Age, years

$$
53 \pm 16 \quad 50 \pm 16 \quad 0.23
$$$$
16(19) \quad 9(17) \quad 0.77
$$

Current smoking, $\mathrm{n}(\%)$

Sicca syndrome-related data

Dry-mouth symptoms, n (\%)

$61(74) \quad 42(78) \quad 0.65$

Major salivary gland swelling, n (\%)

$5(20) \quad 10(63) \quad 0.009$

Positive lip salivary gland biopsy*, n (\%)

$3(13) \quad 16(57) \quad 0.001$

Dry-eye symptoms, n (\%)

$61(74) \quad 41(76) \quad 0.84$

$26(55) \quad 35(83) \quad 0.004$

Positive ocular testst, n (\%)

Xerostomia-related comorbidities

Thyroid gland diseases, n (\%)

$20(24) \quad 12(22) \quad 0.80$

$14(17) \quad 9(17) \quad 0.98$

Organ-specific autoimmune diseases, n (\%)

$7(8) \quad 14(26) \quad 0.005$

Non-SS systemic autoimmune diseases, n (\%)

$48(58) \quad 24(44) \quad 0.13$

Other dry-mouth-related diseasesł, n (\%)

$26(31) \quad 13(24) \quad 0.36$

Dry-mouth-related drugs**, n (\%)

Laboratory data

$\begin{array}{lll}59(73) & 42(86) & 0.087 \\ 9(11) & 26(48) & 0.000 \\ 19(28) & 24(56) & 0.004 \\ 46(56) & 47(87) & 0.000 \\ 4(80) & 2(33) & 0.12 \\ 19(35) & 39(81) & 0.000 \\ 10(18) & 25(52) & 0.000 \\ 8(18) & 4(9) & 0.19\end{array}$

Non-SSA/Ro or SSB/La autoantibodies, n (\%)

SS: Sjögren's syndrome

Erythrocyte sedimentation rate $\geq 14 \mathrm{~mm} / \mathrm{h}, \mathrm{n}$ (\%)

Rheumatoid factor, n (\%)

Hypergammaglobulinemia, n (\%)

Antinuclear antibody, n (\%)

Anti-dsDNA antibody, n (\%)

Anti-SSA/Ro antibody, n (\%)

Anti-SSB/La antibody, n (\%)

*Lip salivary gland biopsy was considered positive if the Chisholm-Mason scale grade $\geq$ III.

†Positive ocular test was considered if the Schirmer I test $\leq 5 \mathrm{~mm}$ and/or pathological ocular staining.

¥Psychogenic, neurological, endocrine, metabolic, infectious, digestive and respiratory disorders.

**Antidepressants, neuroleptics, anxiolytics, antihistamines, opioids, diuretics, antihypertensives and bronchodilators.

Significant $\mathrm{p}$ values are depicted in bold. 
Page 12/15 
Table 2. Differences in salivary gland scintigraphy results among patients with Sjögren's, other autoimmune diseases and other diagnoses

$\begin{array}{ccc}\text { Non-AID } & \text { AID non-SS } & \text { SS } \\ (\mathrm{n}=41) & (\mathrm{n}=42) & (\mathrm{n}=54)\end{array}$

Visual analysis

Abnormal SGS, n (\%)

$\begin{array}{lllll}20(49) & 21(50) & 40(74) & 0.011 & 0.015\end{array}$

$\begin{array}{lllll}14(70) & 19(90) & 31(78) & 0.32 & 0.47\end{array}$

Parotid involvement

$17(85) \quad 18(86) \quad 35(88) \quad 0.41 \quad 0.66$

Submandibular involvement

$17(85) \quad 19(90) \quad 40(100) \quad 0.037 \quad 0.11$

Uptake dysfunction

$\begin{array}{lllll}7(35) & 12(57) & 16(40) & 0.55 & 0.31\end{array}$

Excretion dysfunction

$13(38) \quad 8(28) \quad 27(66) \quad 0.017 \quad 0.002$

Schall's grade $\geq \mathrm{II}, \mathrm{n}(\%)$

$\begin{array}{lllll}7(21) & 5(17) & 25(61) & 0.000 & 0.000\end{array}$

Schall's grade $\geq$ III, n (\%)

$E F \%$

Right parotid gland

Left parotid gland

Parotid mean

Right submandibular gland

Left submandibular gland

Submandibular mean

Overall glands mean

Altered EF\% $(E F \%<50)$

Right parotid gland

Left parotid gland

Right submandibular gland

Left submandibular gland

Semi-quantitative index score

Parotid score (0 to 6 points)

$0(0-4)$

$1(0-4)$

$2(0-4)$

0.46

0.79

Submandibular score (0 to 6 points)

$3(1-5)$

$3(2-5)$

$4(2-6)$

$0.026 \quad 0.40$

Overall glands score (0 to 12 points)

$4(1-8)$

$5(2-9)$

$6(2-9)$

$0.13 \quad 0.51$

AID: autoimmune diseases; EF\%: percentage stimulated excretion fraction; SGS: salivary gland 
scintigraphy; SS: Sjögren's syndrome.

*SS versus AID non-SS, †SS versus Non-AID. U Mann-Whitney for EF\% and semi-quantitative index score, Chi squared for visual analysis and altered EF\%.

Significant $\mathrm{p}$ values are depicted in bold.

ble 3. Association between salivary gland scintigraphy modalities and

I's syndrome related features

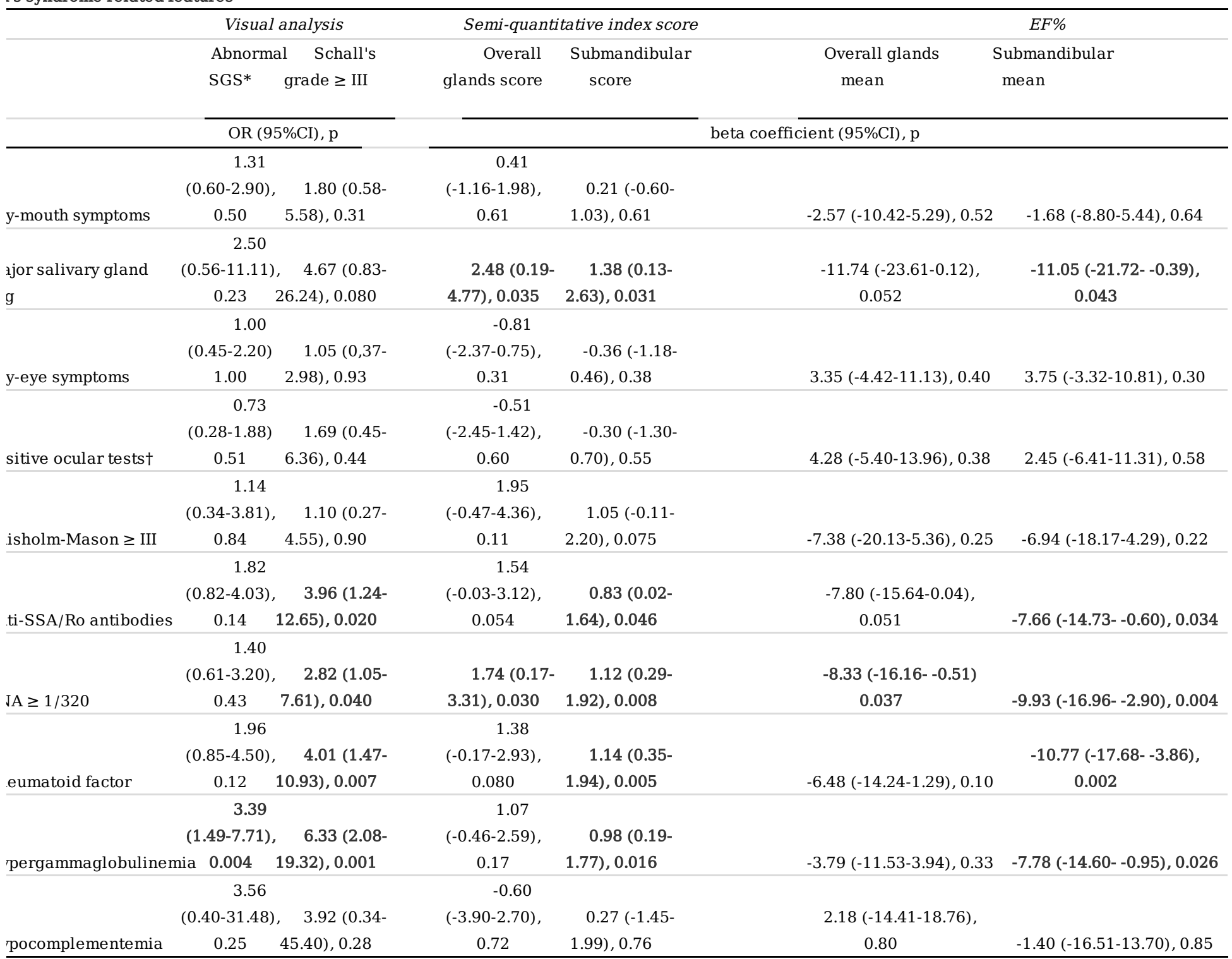

JA: antinuclear antibody; beta coef: beta coefficient; CI: confidence interval; EF\%: percentage stimulated excretion fraction; OR: odds

GS: salivary gland scintigraphy

bnormal salivary gland scintigraphy refers to alterations on tracer uptake, concentration and/or

on in the visual analysis.

ositive ocular test is considered if the Schirmer I test $\leq 5 \mathrm{~mm}$ and/or

gical ocular staining.

gnificant $\mathrm{p}$ values are depicted in bold. 
le 4. Diagnostic discrimination of salivary gland scintigraphy visual lysis and excretion fraction in patients with Sjögren's syndrome

Sjögren's syndrome diagnosis

Clinical AECG Non-SGS ACR judgement criteria* AECG criteria criteria

AUC (95\%CI), p

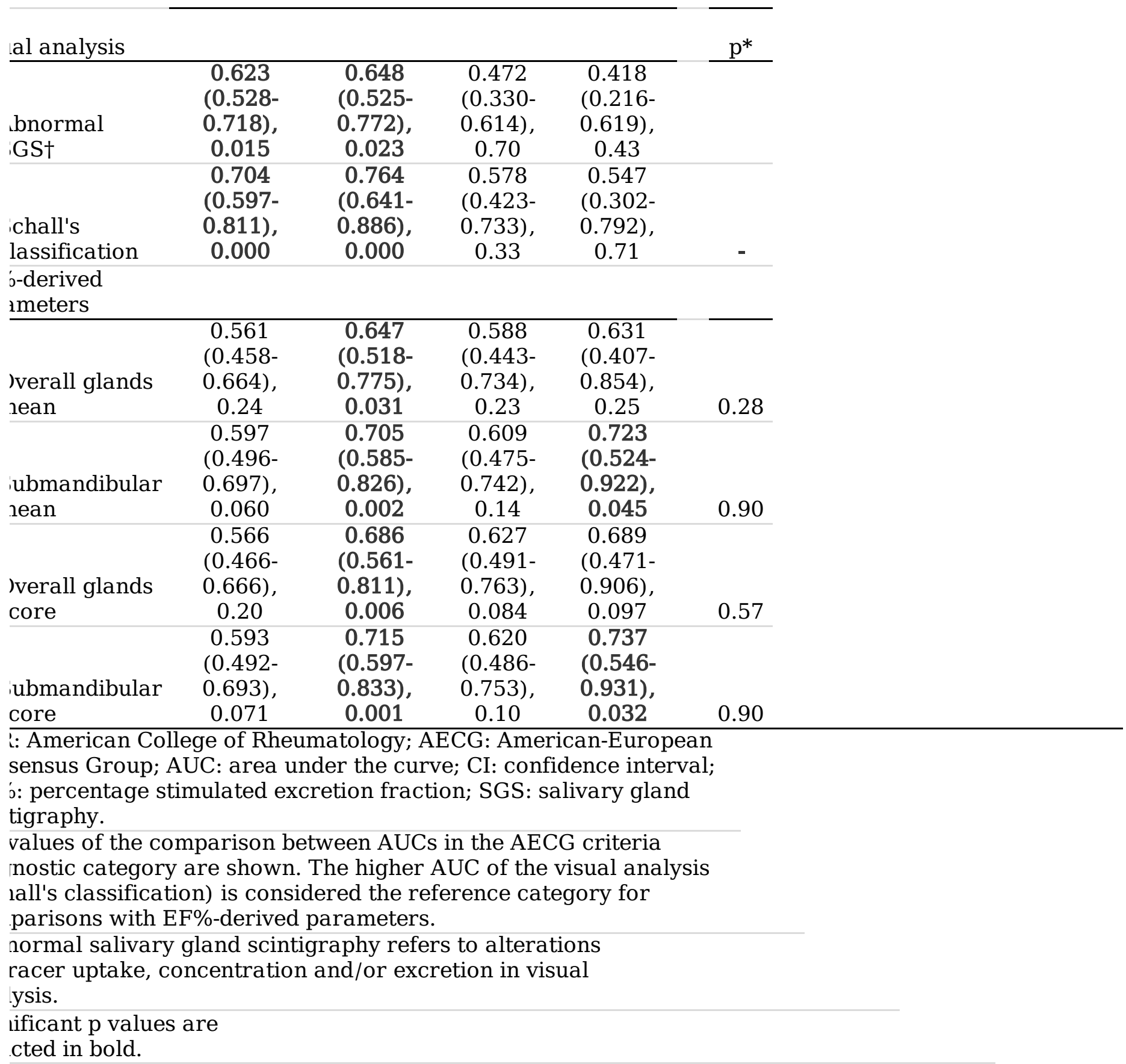

